\title{
AEOLOSAURUS COLHUEHUAPENSIS SP. NOV. (SAUROPODA, TITANOSAURIA) DE LA FORMACIÓN BAJO BARREAL, CRETÁCICO SUPERIOR DE ARGENTINA
}

\author{
GABRIEL CASAL, RUBÉN MARTÍNEZ, MARCELO LUNA \\ Laboratorio de Paleovertebrados, Facultad de Ciencias Naturales, Universidad Nacional de la Patagonia San Juan \\ Bosco, km 4 (9009) Comodoro Rivadavia, Argentina.paleogac@yahoo.com.ar \\ JUAN C. SCIUTTO \\ Facultad de Ciencias Naturales, Departamento de Geología, Universidad Nacional de la Patagonia San Juan Bosco, \\ km 4 (9009) Comodoro Rivadavia, Argentina. \\ MATTHEW LAMANNA \\ Section of Vertebrate Paleontology, Carnegie Museum of Natural History, Pittsburgh, PA, USA.
}

\begin{abstract}
RESUMO - É descrito aqui Aeolosaurus colhuehuapensis sp. nov. com base em uma série caudal articulada proveniente de uma ilha localizada no lago Colhué Huapi, Província de Chubut. Esta série é caracterizada por possuir arcos neurais localizados muito anteriormente em relação ao corpo vertebral e inclinados para frente; prézigapófises bem desenvolvidas projetadas antero-dorsalmente com a presença de facetas articulares amplas em relação as caudais anteriores. Aeolosaurus colhuehuapensis sp. nov. é o único que possui uma profunda e marcada fossa entre o processo transverso e a base da espinha neural nas vértebras caudais mais anteriores; além de possuir uma lâmina delgada que une a base da lâmina pré-espinhal com a parte inferior da faceta articular na parte medial da pré-zigapófises, que por sua vez define uma fossa em ambos os lados da lâmina pré-espinhal; e côndilo posterior em posição central em toda a série caudal. O gênero Aeolosaurus é registrado no CampanianoMaastrichtiano na Argentina e no Brasil, sendo considerado como integrante de uma associação faunística junto com hadrossaurídeos, Madtsoiinae, Chelidae, Sudamericidae e Theria não tribosfénicos encontrados em ambientes de planicies de inundação ou litorais. Sua presença no membro superior da Formacão Bajo Barreal, junto com outros registros paleontológicos da mesma localidade, além de observações geológicas, permitem atribuir preliminarmente uma idade Campaniano-Maastrichtiano? para os níveis superiores desta unidade estratigráfica, aflorantes no sudeste do lago Colhué Huapi, Província de Chubut.
\end{abstract}

Palavras-chave: Titanosauria, Aeolosaurus, Cretáceo Superior, Patagonia, Argentina.

ABSTRACT - AEOLOSAURUS COLHUEHUAPENSIS SP. NOV. (SAUROPODA, TITANOSAURIA), FROM THE BAJO BARREAL FORMATION, UPPER CRETACEOUS OF ARGENTINA. A new sauropod, Aeolosaurus colhuehuapensis. sp. nov., based on an articulated caudal series recovered from an emergent island in Lago Colhué Huapi, Chubut Province is described. This series is characterized by neural arches located very anteriorly above the centrum and inclined forward; well-developed prezygapophyses projecting forward and upward, and wide articular facets in the anterior caudals. Aeolosaurus colhuehuapensis sp. nov. is unique by the presence of a marked and deep fossa between the transverse process and the base of the neural spine in the most anterior caudal vertebrae; a slender lamina that connects the base of the prespinal lamina with the lower part of the articular facet in the medial part of the prezygapophysis, defining a fossa on both sides of the prespinal lamina; posterior condyle centrally placed in the entire caudal series. The genus Aeolosaurus is recorded in the Campanian-Maastrichtian in Argentina and Brazil, and is considered part of a faunal association with hadrosaurids, Madtsoiinae, Chelidae, Sudamericidae, and non-tribosphenic Theria in floodplain or littoral environments. Its presence in the Upper Member of the Bajo Barreal Formation, along with other paleontological records from the same locality, and geologic observations allow us to assign preliminarily a Campanian-Maastrichtian? age for the upper levels of this stratigraphic unit outcropping in the southeast of Lago Colhué Huapi, Chubut Province.

Key words: Titanosauria, Aeolosaurus, Upper Cretaceous, Patagonia, Argentina. 


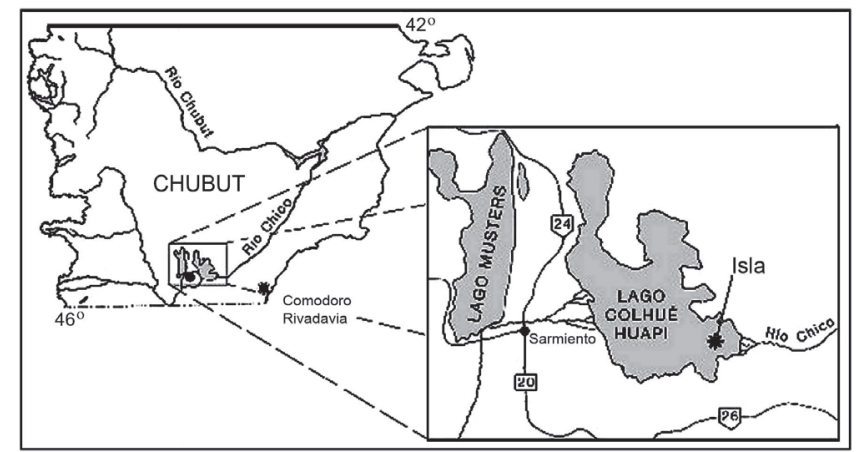

Figura 1. Mapa de ubicación geográfica.

Figure 1. Location map of the collecting site.

\section{INTRODUCCIÓN}

El hallazgo de los materiales que se describen en este trabajo, consistente en una serie de vértebras caudales articuladas de un titanosaurio, fue efectuado por un grupo de pescadores, y denunciado al Laboratorio de Paleontología de Vertebrados de la Universidad Nacional de la Patagonia San Juan Bosco, en Comodoro Rivadavia. La extracción y estudio de esta serie caudal se realizó en el marco del proyecto de investigación (Los vertebrados del Grupo Chubut: características y evolución), realizándose en el Lago Colhué Huapi, ubicado en el centro-sur de la provincia de Chubut. En este lago el nivel de cota ha variado notablemente durante los últimos años, desde su desecación prácticamente total en el 2000, hasta un importante aumento en el nivel de sus aguas en el 2005. En estadios intermedios quedaron expuestas algunas islas de no más de un metro de desnivel y algunos centenares de metros de extensión. Las mismas están constituidas por sedimentitas continentales del Cretácico Superior asignadas al miembro superior de la Formación Bajo Barreal, unidad perteneciente al Grupo Chubut (Sciutto et $a l$., en prensa).

Las condiciones extremas de humedad y sequía a las que estuvieron sometidos los materiales incidieron directamente sobre su estado de preservación. Sin embargo, el hallazgo posee aún un especial interés, debido al aporte que brinda para el conocimiento del registro y distribución de los titanosaurios de Patagonia. En la misma campaña se recolectaron, entre otros, restos desarticulados de ornitópodos (Luna et al., 2003; Ibiricu, 2004) y las mandíbulas incompletas de un cocodriliforme (Lamanna et al., 2003).

Los titanosaurios son los saurópodos cretácicos mejor representados en Sudamérica, especialmente en Patagonia (Bonaparte, 1986; Salgado \& Coria, 1993), siendo numerosos los hallazgos en el Sur de la provincia de Chubut, en afloramientos de la Formación Bajo Barreal. En esta unidad es posible diferenciar, a partir de la estratigrafía y los vertebrados fósiles, una fauna de dinosaurios para el Cretácico Superior temprano (Cenomaniano-Turoniano) y otra para el Cretácico Superior tardío (Campaniano-Maastrichtiano?) (Casal et al., 2006). La primera está representada en la Ea. Ocho Hermanos, en el anticlinal Sierra San Bernardo, que ha brindado el titanosaurio basal Epachthosaurus sciuttoi Martínez et al. (2004) y la Ea. Laguna Palacios, en el anticlinal Sierra Nevada, que ha aportado el cráneo de un saurópodo titanosauriforme (Martínez, 1998, 2006) junto a numerosos restos articulados y aislados asignados a estos saurópodos. Por su parte el titanosaurio, objeto de este trabajo, procede de niveles más tardíos, probablemente del CampanianoMaastrichtiano? de afloramientos expuestos en el Sudeste del Lago Colhué Huapi y río Chico.

Abreviaturas institucionales. UNPSJB-PV, paleontología de vertebrados, Universidad Nacional de la Patagonia San Juan Bosco, Comodoro Rivadavia; MPMA, Museo de Paleontología, Centro Cultural, Monte Alto; CPP, Centro de Pesquisas Paleontológicas Llewellyn Ivor Price, Uberaba; MG: MP, Museu de Paleontología da Universidade Federal do Mato Grosso; MPCA, Museo Provincial Carlos Ameghino, Cipolletti, Río Negro; MACN-RN, Museo Argentino de Ciencias Naturales Bernardino Rivadavia, colección Río Negro, Buenos Aires.

\section{MARCO GEOLÓGICO REGIONAL}

Formación Bajo Barreal (Senoniano). Esta unidad tiene una amplia distribución tanto en la Sierra de San Bernardo como en el resto de la cuenca. Se presenta conformando lomadas redondeadas, a veces del tipo badlands, en bancos medianos a gruesos, bien estratificados y semicubiertos por derrubios basálticos o por minerales arcillosos hidratables, producidos por meteorización de materiales primariamente piroclásticos. Su espesor es variable desde unos 150 metros al norte de la Laguna Palacios hasta más de 1.000 metros en el Codo del Río Senguerr (Sciutto, 1981).

En el Sur-Este del lago Colhué Huapi aflora el miembro superior de la Formación Bajo Barreal que es de baja consolidación relativa, y aparece normalmente cubierto por una costra de meteorización arcillosa. El material piroclástico es progresivamente reemplazado por sedimentos cada vez más fangolíticos que corresponden a depósitos fluviales meandrosos. Hacia el este y sur-este de la zona de afloramientos los constituyentes de esta Formación se van haciendo cada vez más epiclásticos, con predominio de areniscas y pelitas y ausencia casi total de tobas (Sciutto et al., en prensa).

Formación Salamanca (Paleoceno inferior). Esta unidad marina se deposita en discordancia erosiva regional sobre la Formación Bajo Barreal, o bien como sucede en ambas márgenes del valle del Río Chico, sobre un manto de roca básica. Tiene un espesor bastante uniforme y se va incrementando suavemente desde el oeste (Codo del Río Senguerr) hasta alcanzar los 300 metros en el golfo San Jorge. Está compuesta fundamentalmente por areniscas y coquinas verdosas en su base, fangolitas gris verdosas en los términos medios y areniscas verde oliva y fangolitas grises en su tope. En general, sus depósitos se consideran pertenecientes a un ambiente marino proximal, poco profundo y en su mayoría de elevada energía, especialmente hacia el oeste donde se incrementa considerablemente la proporción areniscosa. 
Aunque en su tope también se han descrito depósitos de baja energía, del tipo albuféricos.

Las areniscas glauconíticas inferiores (miembro glauconítico) representan a un ambiente marino proximal de barras costeras, producidas durante la transgresión marina cretácico-terciaria, con un marcado diacronismo, siendo progresivamente más jóvenes hacia el oeste. Feruglio (1949), ya reconoce que sus sedimentos constituyen originalmente un manto continuo, cuyo espesor decrece progresivamente hacia el oeste y transgrediendo sobre una superficie de erosión, en general, sobre el Grupo Chubut, pero guardando con él un cierto paralelismo, por lo menos en cortas distancias. En el valle superior del Río Chico la unidad se apoya sobre una colada basáltica (Feruglio, 1949; Sciutto et al., en prensa).

\section{PALEONTOLOGÍA SISTEMÁTICA}

\author{
SAURISCHIA Seeley, 1887 \\ SAUROPODOMORPHA von Huene, 1932 \\ SAUROPODA Marsh, 1878 \\ NEOSAUROPODA Bonaparte, 1986 \\ TITANOSAURIFORMES Salgado et al., 1997 \\ TITANOSAURIA Bonaparte \& Coria, 1993 \\ AEOLOSAURINI Franco-Rosas et al., 2004 \\ Aeolosaurus Powell, 1987
}

Especies dentro del género. Aeolosaurus rionegrinus Powell, 1987 y la nueva especie descrita aquí.

Diagnosis modificada para el género. Saurópodo titanosaurio de tamaño medio que posee los siguientes caracteres: vértebras caudales medias con las postzigapófisis ubicadas en posición muy anterior respecto del cuerpo vertebral alcanzando el borde articular anterior; y hemapófisis abiertas con facetas articulares dispuestas en dos planos.

\section{Aeolosaurus colhuehuapensis sp. nov.} (Figuras 2-6)

Diagnosis. Se distingue de A. rionegrinus por la presencia en vértebras caudales anteriores de profundas fosas entre los procesos transversos y la base de la espina neural; presencia de una delgada lámina que une la base de la lámina prespinal y el borde inferior de la faceta articular en la parte medial de las prezigapófisis, que define una fosa a ambos lados de la lámina prespinal; vértebras caudales con el cono articular posterior en posición media.

Diagnosis. A. colhuehuapensis is distinguished from A. rionegrinus by the following characters: anterior caudal vertebrae possessing deep fossae between the transverse processes and the base of the neural spine; anterior caudal vertebrae with a slender lamina joining the base of the prespinal lamina with the lower edge of the articular facet in the medial part of the prezygapophysis, producing a fossa on both sides of the prespinal lamina; caudal vertebrae with the posterior articular cone centrally placed.
Holotipo. UNPSJB-PV 959/1 a UNPSJB-PV 959/27, 21 vértebras caudales articuladas y siete hemapófisis articuladas (Figura 2).

Etimología. colhuehuapensis se refiere al Lago Colhué Huapi, ubicado en el centro-Sur de la Provincia de Chubut, sitio en el que se realizó el hallazgo.

Procedencia geográfica. Isla emergida al Sur-Este del Lago Colhué Huapi (4535'52"S y 68 37'20”W), Departamento Sarmiento, Provincia del Chubut, Argentina (Figura 1).

Procedencia estratigráfica. Miembro superior de la Formación Bajo Barreal, Grupo Chubut. La serie caudal se hallaba incluida en areniscas tobáceas de color gris a castaño claro.

Edad. Cretácico Superior (Senoniano).

Descripción. Las vértebras caudales anteriores (Figura 3A y 3B) son fuertemente procélicas, presentan el cuerpo vertebral corto y el cono posterior en posición central. Sobre una de las caras laterales del cuerpo caudal más anterior UNPSJBPV 959/1 (caudal tercera) hay una depresión notoria aunque no llega a ser un pleurocelo definido. La cara ventral es relativamente ancha. En esta vértebra, el arco neural se encuentra separado del centro por efectos de la preservación. Los procesos transversos son relativamente largos comparados con otros titanosaurios, y se encuentran proyectados lateroposteriormente. Las prezigapófisis son robustas, proyectadas anterodorsalmente con un ángulo aproximado de $50^{\circ}$ respecto del plano horizontal, y con facetas articulares muy amplias de contorno ligeramente oval. Las postzigapófisis ubicadas sobre la mitad anterior del cuerpo vertebral, por encima del canal neural, están incompletas, pero muestran un buen desarrollo en sentido dorsoventral y se orientan lateroventralmente. De la espina neural solo se conservó la base y parte de la lámina prespinal; que de acuerdo a su disposición probablemente haya estado inclinada anteriormente. Entre el proceso transverso y la base de la espina neural existe una marcada y profunda oquedad, carácter observado desde la tercera a la quinta vértebra caudal. En la cara medial de las prezigapófisis hay también una fosa a ambos lados de la lámina prespinal limitada por una delgada lámina que conecta la base de la lámina prespinal con el borde inferior de la faceta articular, llamada aquí lámina infraespinoprezigapofisial. La lámina espinoprezigapofisial es muy gruesa (Figura 3B).

Las vértebras quinta, séptima y octava son fuertemente procélicas, con centros cortos y caras laterales altas y levemente cóncavas en sentido anteroposterior. Los procesos transversos son largos y dirigidos posteriormente y levemente en sentido ventral. El arco neural está ubicado en la porción más anterior del centro, está inclinado hacia adelante y ocupa prácticamente la mitad anterior del centro. Lo que se ha conservado de las prezigapófisis indica que estaban proyectadas anterodorsalmente.

Las vértebras caudales medias en vista lateral presentan el margen anterior del cuerpo inclinado hacia adelante (Figura 3C). En la vértebra caudal novena, UNPSJB-PV 959/11, el cuerpo vertebral presenta un desarrollo de la cara lateral secundaria como en Salgado \& García, 2002, por lo que en 


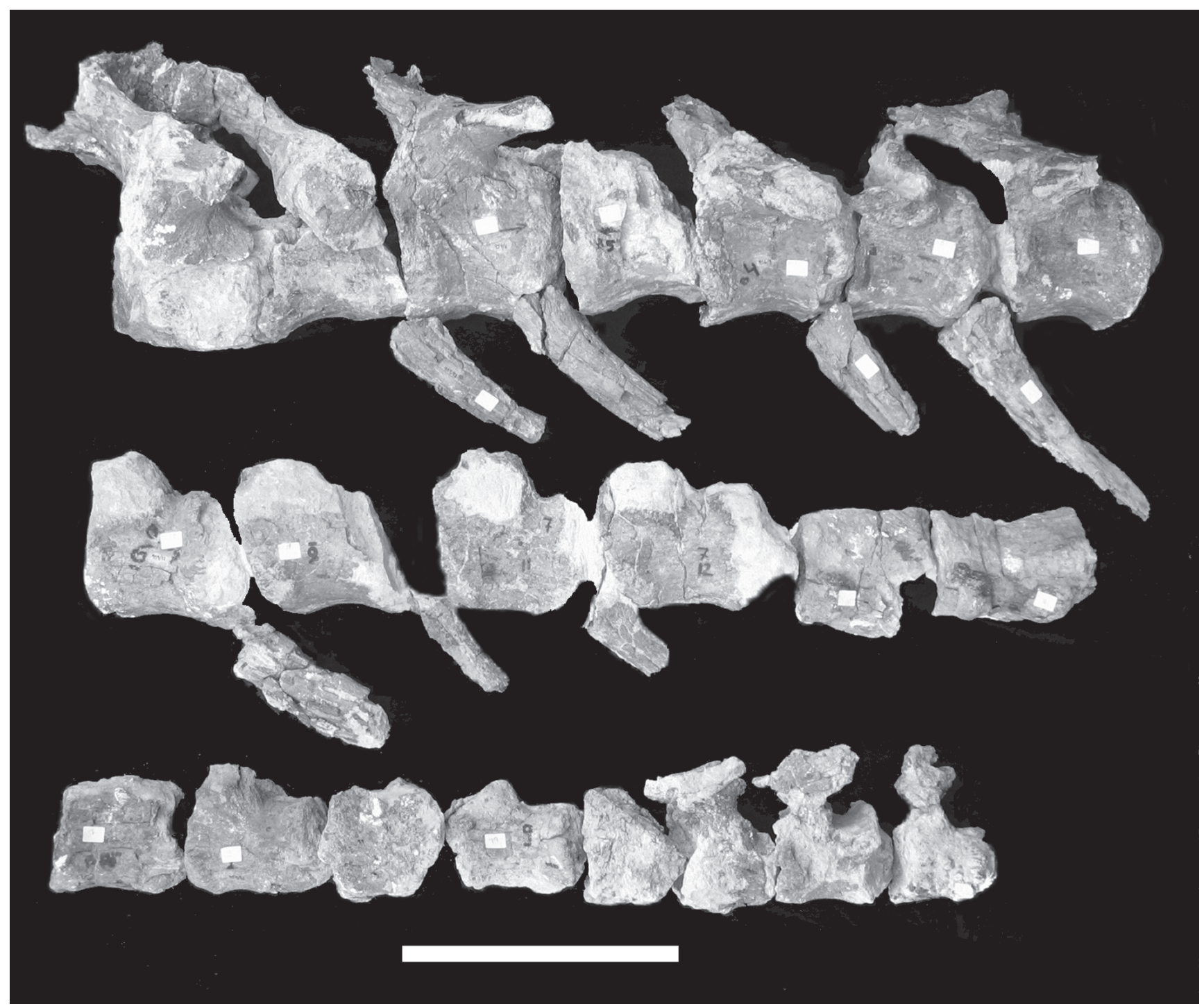

Figura 2. Holotipo de Aeolosaurus colhuehuapensis nov. sp. UNPSJB PV 959 en vista lateral izquierda. Escala= $30 \mathrm{~cm}$. Figure 2. Holotype of Aeolosaurus colhuehuapensis nov. sp. UNPSJB PV 959 in left lateral view. Scale bar= $30 \mathrm{~cm}$.

vista posterior el cuerpo adopta un contorno hexagonal (Figura 3D). El cono articular posterior es prominente y se ubica en posición central. La cara ventral es angosta y posee dos protuberancias notorias en la parte posterior para la articulación de las hemapófisis. El arco neural de la novena caudal está ubicado en la parte anterior del centro y está inclinado hacia adelante. Las prezigapófisis están dirigidas horizontalmente, son muy largas y alcanzan el borde anterior del cuerpo vertebral de la vértebra que le precede, es decir, que las prezigapófisis son casi tan largas como el cuerpo vertebral. Las postzigapófisis se ubican también muy anteriormente, por encima del borde anterior del cuerpo, son de contorno ovalado y con el eje mayor orientado en sentido dorso-ventral.

El estado de conservación del resto de las vértebras caudales medias es bastante malo, pero es posible observar que los centros poseen características similares a las descritas. Son cortos, con caras laterales altas, y cara ventral relativamente angosta. En los casos en que se ha preservado parte del arco neural, éste está ubicado muy anteriormente, más adelante que en las caudales anteriores, y ocupa la mitad anterior del cuerpo vertebral.

Las vértebras caudales distales 21, 22 y 23 (UNPSJB-PV 959/25-27; Figura 4) presentan un centro corto y los procesos transversos se encuentran reducidos a una cresta horizontal. El arco neural es corto y alto, ubicado sobre el margen anterior del cuerpo vertebral, y dirigido verticalmente respecto de éste. Las prezigapófisis se conservaron parcialmente, pero es evidente que se proyectaban horizontalmente. Las postzigapófisis se desarrollan con una inclinación posteroventral, con superficies articulares amplias y ubicadas sobre la mitad del cuerpo.

Se han recuperado siete hemapófisis articuladas en vértebras caudales anteriores y medias. Presentan extremos proximales separados, con superficies articulares amplias dispuestas en dos planos bien definidos en ángulo (Figura 5). 


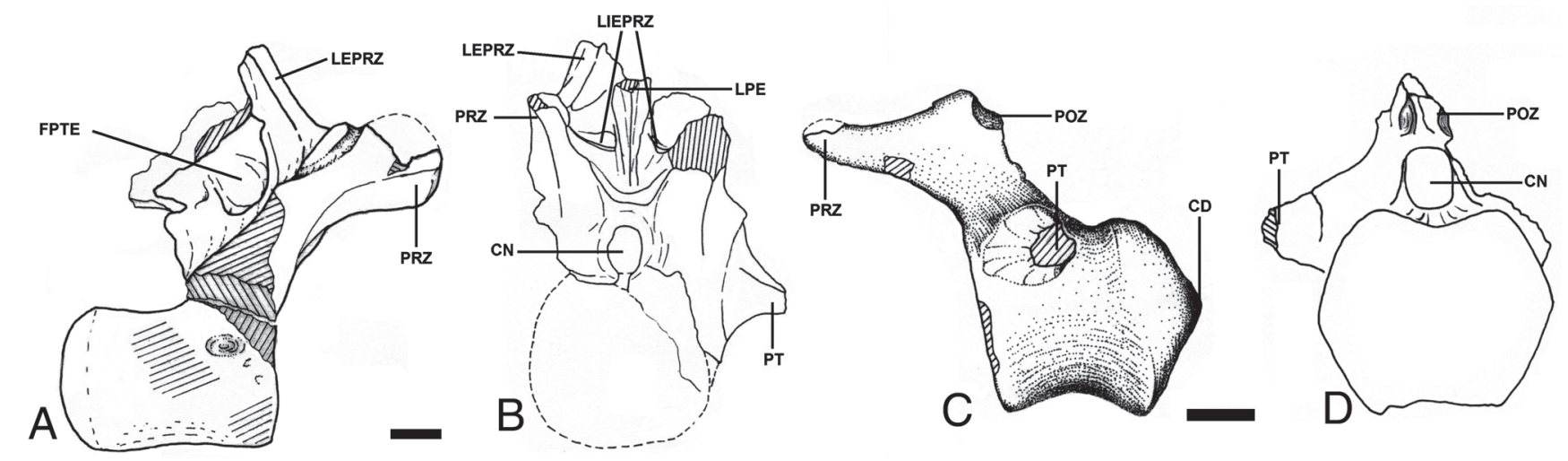

Figura 3. Aeolosaurus colhuehuapensis nov. sp.: UNPSJB PV 959/1, tercera? vértebra caudal en vista lateral derecha (A) y en anterior (B); UNPSJB-PV 959/11, novena? vértebra caudal en vista lateral izquierda (C) y posterior (D). Abreviaturas: CN, canal neural; PRZ, prezigapófisis; PT, proceso transverso; POZ, postzigapófisis; LPE, lámina prespinal; LEPRZ, lámina espinoprezigapofisial; LIEPRZ, lámina infraespinoprezigapofisial; FPTE, fosa entre el proceso transverso y la espina neural; CD, cóndilo. Escala $=5 \mathrm{~cm}$.

Figure 3. Aeolosaurus colhuehuapensis nov. sp.: UNPSJB-PV 959/1, third? caudal vertebra in right lateral (A) and anterior (B) views; UNPSJB-PV 959/11, ninth? caudal vertebra in left lateral (C) and posterior (D) views. Abbreviations: CN, neural canal; PRZ, prezygapophysis; PT, transverse process; POZ, postzygapophysis; LPE, prespinal lamina; LEPRZ, spinoprezygapophyseal lamina; LIEPRZ, infraspinoprezygapophyseal lamina; FPTE, fossa between transverse process and neural spine; CD, condyle. Scale bar $=5 \mathrm{~cm}$.

\section{COMPARACIONES}

Las comparaciones se efectuaron con varios géneros cretácicos de titanosaurios, incluyendo formas basales como Andesaurus y Epachthosaurus, y saurópodos más derivados incluyendo a los registros documentados dentro de Aeolosaurini (Franco-Rosas et al., 2004). Para la ubicación anatómica de los materiales UNPSJB-PV 959/1 a 959/27 se tuvieron en cuenta las secuencias caudales de los géneros utilizados en la comparación.

\section{Comparación con titanosaurios no Aeolosaurini}

El ejemplar del lago Colhué Huapi tiene las prezigapófisis de las vértebras caudales anteriores más largas que las de Baurutitan britoi Kellner, Campos \& Trotta (2005) y mucho más que las de E. sciuttoi. Las facetas articulares de las prezigapófisis también son más amplias. En las caudales medias de A. colhuehuapensis sp. nov. el arco neural se ubica muy anteriormente y está inclinado hacia adelante, lo que no ocurre en Andesaurus delgadoi Calvo \& Bonaparte (1991), Epachthosaurus ni en Baurutitan. En la caudal media novena las prezigapófisis son mucho más largas que en éstos y otros titanosaurios. Asimismo, las postzigapófisis se ubican en la mitad anterior del cuerpo vertebral, mientras que en Andesaurus, Baurutitan, Epachthosaurus y Alamosaurus sanjuanensis Gilmore (1946) se ubican hacia la mitad posterior (Figura 6). A diferencia de estos titanosaurios, los arcos neurales de las vértebras caudales distales en la serie caudal de A. colhuehuapensis sp.nov. se ubican muy anteriormente, sobre el borde anterior del centro.

Los arcos hemales presentan superficies articulares con dos planos definidos en diferentes ángulos, mientras que los arcos hemales de Andesaurus y Epachthosaurus presentan superficies articulares con un solo plano.

\section{Comparación dentro de Aeolosaurini}

Este clado incluye a Aeolosaurus rionegrinus, Gondwanatitan faustoi Kellner \& Azevedo (1999) y Rinconsaurus caudamirus Calvo \& Gonzalez Riga (2003). Otros materiales referidos a Aeolosaurini son: Aeolosaurus sp. MPMA/ sin número (Santucci \& Bertini, 2001); Aeolosaurus sp. CPP 298 (Santucci \& Bertini, 2001); Aeolosaurus sp. MPCA 27100 (Salgado et al., 1997) y MPCA 27174 (Salgado \& Coria, 1993); Aeolosaurus rionegrinus? MACN-RN 107 (Powell, 1987); Gondwanatitan sp. MP 284288 (Franco-Rosas et al., 2004).

Aeolosaurus colhuehuapensis sp. nov. comparte con $A$. rionegrinus, $R$. caudamirus, Gondwanatitan faustoi y Aeolosaurus sp. (Salgado \& Coria, 1993; Salgado et al., 1997) una marcada procelia en las caudales anteriores. Aeolosaurus colhuehuapensis sp. nov. también comparte con estos géneros la presencia de cuerpo vertebral corto con caras ventrales anchas, caras laterales altas y suavemente cóncavas en sentido anteroposterior. Sobre la cara lateral de la tercera caudal de $A$. colhuehuapensis sp. nov. hay una depresión notoria como en A. rionegrinus, Gondwanatitan faustoi y Rinconsaurus caudamirus que no llega a ser un pleurocelo definido.

Las caudales anteriores de la serie de Aeolosaurus colhuehuapensis sp. nov. (Figura 3A), comparten el siguiente carácter sinapomórfico de Aeolosaurini: prezigapófisis largas, proyectadas anterodorsalmente y con amplias facetas articulares. Como en Aeolosaurus sp. de la Formación Los Alamitos (Salgado et al., 1997), sobre el borde dorsal de las prezigapófisis de A. colhuehuapensis sp. nov., existe un marcado surco pero que no llega a formar facetas articulares bilobuladas como en el ejemplar de la Provincia de Río Negro. Las postzigapófisis de A. colhuehuapensis sp. nov., A. rionegrinus y Aeolosaurus sp. (Salgado \& Coria, 1993) tienen 


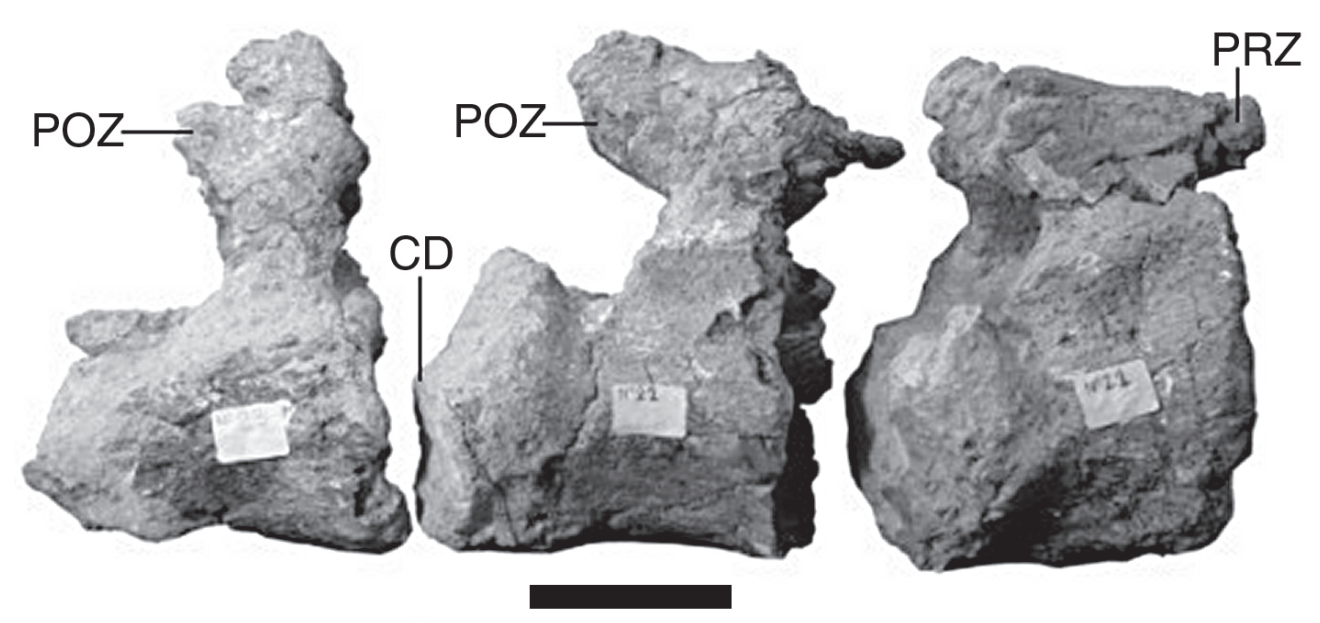

Figura 4. Aeolosaurus colhuehuapensis nov. sp. UNPSJB-PV 959/25-27. Vértebras caudales distales 21-22 y 23? en vista lateral derecha. Abreviaturas: PRZ, prezigapófisis; POZ, postzigapófisis; CD, cóndilo. . Escala $=5 \mathrm{~cm}$.

Figure 4. Aeolosaurus colhuehuapensis nov. sp., UNPSJB-PV 959/25-27. Distal caudal vertebrae $21^{\text {st }}, 22^{\text {nd }}$ and $23^{\text {rd }}$ ? in right lateral view. Abbreviations: PRZ, prezygapophysis; POZ, postzygapophysis; CD, condyle.

Scale bar $=5 \mathrm{~cm}$.

similar desarrollo y ubicación respecto del cuerpo vertebral. Sin embargo, en A. rionegrinus son casi paralelas al plano sagital, mientras que en $A$. colhuehuapensis sp. nov. y Aeolosaurus sp. de la Formación Allen (Salgado \& Coria, 1993) se orientan lateroventralmente con una mayor inclinación respecto de dicho plano. Entre el proceso transverso y la base de la espina neural de las caudales anteriores de $A$. colhuehuapensis sp. nov. existe una marcada y profunda fosa, carácter observado, al menos, desde la tercera a la quinta vértebra caudal. En la cara medial de las prezigapófisis se desarrolla una delgada lámina que conecta la parte inferior de las prezigapófisis con la base de la lámina prespinal. Esta lamina infraespinoprezigapofisial define dos fosas a ambos lados de la lámina prespinal (Figura 3B). Estos últimos dos caracteres no están presentes en ningún Aeolosaurini ni en otros titanosaurios y son, por lo tanto, considerados autapomorfías de Aeolosaurus colhuehuapensis sp. nov..

Las caudales medias de A. colhuehuapensis sp. nov., particularmente la caudal novena (Figura 3C), presentan cara ventral angosta con marcadas prominencias para la articulación de las hemapófisis como en Aeolosaurus sp. (Salgado \& Coria, 1993). El margen anterior del cuerpo vertebral esta inclinado hacia delante, carácter considerado sinapomórfico de Aeolosaurini. Asimismo, la caudal novena del sur de Chubut presenta un desarrollo de la cara lateral secundaria que en vista posterior le otorga un contorno hexagonal similar a lo observado en la quinta caudal de Gondwanatitan (Figura 3D). El arco neural se ubica muy anteriormente y está inclinado hacia adelante como en todos los Aeolosaurini.

En las caudales medias de A. colhuehuapensis sp. nov. las prezigapófisis son algo más cortas que en $A$. rionegrinus pero más largas que en Aeolosaurus sp. (Salgado \& Coria, 1993). Las postzigapófisis de A. colhuehuapensis sp. nov., $A$. rionegrinus y Aeolosaurus sp. (Salgado \& Coria, 1993) se ubican en la parte más anterior respecto del centro, alcanzando el borde articular anterior del cuerpo vertebral, una posición mucho más avanzada que en otros titanosaurios (Figura 3C).

Las caudales distales de A. colhuehuapensis sp. nov. son similares en su morfología general a las de Gondwanatitan, pero en el ejemplar de Chubut el arco neural es más alto y se ubica más anteriormente respecto del cuerpo vertebral. Ambos comparten la disposición de las postzigapófisis y los procesos transversos reducidos a una cresta horizontal.

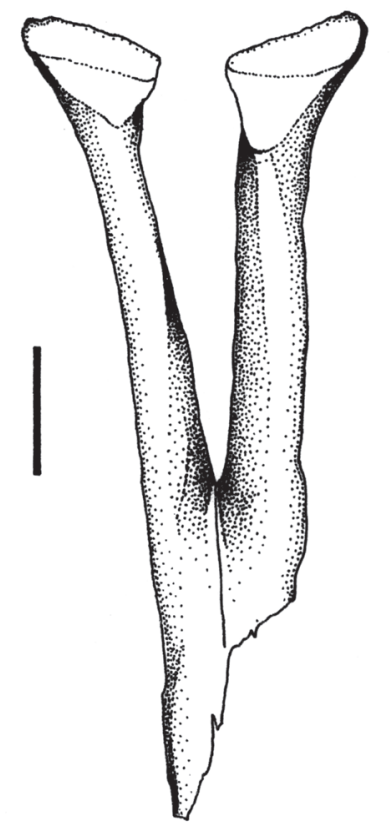

Figura 5. Aeolosaurus colhuehuapensis sp. nov., UNPSJB-PV 959/5, hemapófisis de caudal media en vista posterior. Escala $=5$ $\mathrm{cm}$.

Figure 5. Aeolosaurus colhuehuapensis sp. nov., UNPSJB-PV $959 / 5$, a middle caudal haemal arch in posterior view. Scale bar $=$ $5 \mathrm{~cm}$. 

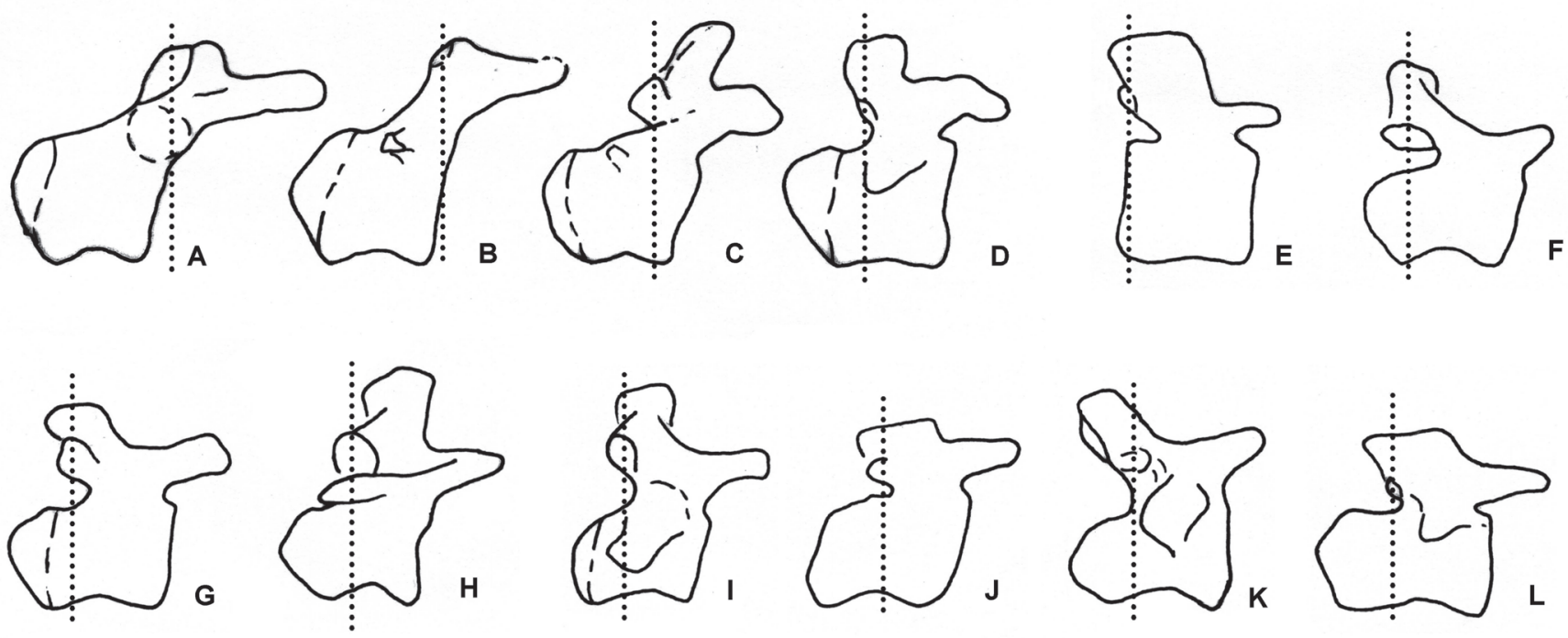

Figura 6. Ubicación de las postzigapófisis respecto del centro vertebral en vértebras caudales medias proximales en distintos titanosaurios. A, A. rionegrinus; B, A. colhuehuapensis sp. nov. (UNPSJB-PV 959/11); C, Aeolosaurus sp.; D, Alamosaurus sanjuanensis; E, Andesaurus delgadoi; F, Neuquensaurus australis; G, Baurutitan brito; H, Titanosaurinae indet.; I, Titanosaurus sp.; J, Mendozasaurus neguyelap; K, Saltasaurus Ioricatus; L, Epachthosaurus sciuttoi. Modificado de Powell, 2003 (A, F, H, I, K);Salgado \& Coria, 1993 (C); Gilmore, 1946 (D); Calvo \& Bonaparte, 1991 (E); Kellner et al., 2005 (G); González Riga, 2003 (J); y Martínez et al., 2004 (L).

Figure 7. Location of the postzygapophyses referring to vertebral centrum in proximal middle caudals of different titanosaurs. A, $A$. rionegrinus; B, A. colhuehuapensis sp. nov. (UNPSJB-PV 959/11); C, Aeolosaurus sp.; D, Alamosaurus sanjuanensis; E, Andesaurus delgadoi; F, Neuquensaurus australis; G, Baurutitan britoi; H, Titanosaurinae indet.; I, Titanosaurus sp.; J, Mendozasaurus neguyelap; K, Saltasaurus loricatus; L, Epachthosaurus sciuttoi. Modified from Powell, 2003 (A, F, H, I, K); Salgado \& Coria, 1993 (C); Gilmore, 1946 (D); Calvo \& Bonaparte, 1991 (E); Kellner et al., 2005 (G); González Riga, 2003 (J); and Martínez et al., 2004 (L).

En toda la serie caudal de $A$. colhuehuapensis sp. nov. el cono articular posterior se ubica en posición media, mientras en A. rionegrinus y en Aeolosaurus sp. (Salgado \& Coria, 1993; Salgado et al., 1997) está desplazado dorsalmente.

Las hemapófisis de $A$. colhuehuapensis sp. nov. y $A$. rionegrinus son similares con extremos proximales abiertos y con superficies articulares dobles definiendo dos planos en diferente ángulo.

\section{DISCUSIÓN}

El clado Aeolosaurini fue descrito por Franco-Rosas et al. (2004) en base a los siguientes caracteres: cuerpos caudales anteriores y medios con el margen anterior inclinado hacia adelante; arcos neurales dispuestos sobre el borde anterior del centro en las vértebras caudales medias; espina neural inclinada hacia adelante al menos en las caudales medias; prezigapófisis alargadas en las caudales anteriores y medias, en correlación con el desplazamiento del arco neural hacia adelante; facetas articulares de las prezigapófisis y postzigapófisis alargadas anteroposteriormente, al menos en las caudales anteriores y medias. Incluye a A. rionegrinus, $R$. caudamirus, G. faustoi.

Por su parte, A. colhuehuapensis sp. nov. comparte estos caracteres sinapomórficos del clado que permiten considerarlo como un nuevo integrante de los Aeolosaurini.
Asimismo, A. colhuehuapensis sp. nov. exhibe claramente caracteres reconocidos en el género Aeolosaurus. El carácter referido a las vértebras caudales medias con las postzigapófisis ubicadas en posición muy anterior respecto del cuerpo vertebral, alcanzando el borde articular, puede observarse claramente en A. rionegrinus y Aeolosaurus sp. (Salgado \& Coria, 1993; Figura 6). Sin embargo este carácter no está presente en otros Aeolosaurini como Rinconsaurus y Gondwanatitan, y tampoco en otros titanosaurios. Esto permite proponer al carácter mencionado como sinapomorfía de Aeolosaurus.

El carácter hemapófisis abiertas con facetas articulares dispuestas en dos planos constituye un rasgo mencionado por Powell (1987) en la descripción original de $A$. rionegrinus y por Salgado \& Coria (1993), pero omitido por Salgado et al. (1997). La presencia de este carácter en $A$. colhuehuapensis sp. nov. nos lleva a proponerlo como diagnóstico del género.

En las vértebras caudales distales del ejemplar del sur de Chubut, el arco neural es corto y alto, ubicado sobre el margen anterior del cuerpo vertebral. Consecuentemente, es posible pensar que el arco neural se ubicaría anteriormente a lo largo de toda la serie caudal de Aeolosaurini ya que en las caudales distales de G. faustoi y Aeolosaurus sp. este carácter también es observado (Salgado \& Coria, 1993; Kellner \& Azevedo, 1999). La disposición del arco neural en las caudales distales de A. colhuehuapensis sp. nov. y G. faustoi, sugiere que en 
este tramo de la secuencia caudal de los Aeolosaurini, el arco neural ya no esta inclinado hacia adelante, sino que se proyecta verticalmente. Por lo tanto, el carácter arco neural ubicado muy anteriormente y dirigido verticalmente en las caudales distales, podría ser una sinapomorfía de Aeolosaurini.

Por otra parte los rasgos específicos de $A$. colhuehuapensis sp. nov., diferenciales en relación a todos los registros documentados dentro de Aeolosaurini, son evidentes. En relación a la lámina conectora entre la parte inferior de las prezigapófisis y la base de la lámina prespinal, que define una fosa, se trata de un rasgo que podría estar relacionado con la observación de Salgado et al. (2006) sobre la posible presencia, en las caudales anteriores de algunos titanosaurios, de divertículos aéreos a ambos lados de la lámina prespinal. La fosa presente entre los procesos transversos y la base de la espina neural, podría cumplir la función de inserción muscular.

El género Aeolosaurus está registrado a partir del Campaniano-Maastrichtiano en las Formaciones Allen (Salgado \& Coria, 1993), Los Alamitos (Salgado et al., 1997) y Angostura Colorada (Powell, 1987), en la provincia de Río Negro, en la Argentina. En Brasil este género se registra en la Formación Adamantina en el Estado de São Paulo (Bertini et al., 1999a) y en la base del miembro Serra Galga de la Formación Marília en el Estado de Minas Gerais (Bertini et al., 1999b) ambas asignadas al Campaniano tardíoMaastrichtiano temprano. Estratigráficamente, $A$. colhuehuapensis sp. nov. proviene de niveles próximos a la superficie discordante que separa el Cretácico del Terciario. Esta superficie no habría eliminado un espesor considerable del Cretácico, ya que los sedimentos se mantienen casi horizontales por encima y debajo de ella, por lo que el hiatus involucrado no representaría muchos millones de años. Por ello, es lógico pensar en un pasaje desde un Cretácico muy joven (Campaniano-Maastrichtiano?) a un Terciario muy antiguo (Paleoceno inferior).

Desde el punto de vista paleoambiental, las observaciones geológicas realizadas en los afloramientos de la Formación Bajo Barreal en el SE de lago Colhué Huapi y nacientes del río Chico, permiten interpretar un paleoambiente de amplias planicies de inundación y con cursos fluviales meandriformes de alta sinuosidad próximos a su desembocadura en un cuerpo de agua mayor. Se trataría de un ambiente similar al que se define para la asociación faunística que integra Aeolosaurus junto a hadrosáuridos, Madtsoiinae, Chelidae, Sudamericidae y Theria no tribosfénicos en Formación Los Alamitos. En esta asociación se basó Bonaparte $(1986,1992)$ para definir la edad biocronológica denominada edadvertebrado Alamitense, asignada al Campaniano tardíoMaastrichtiano temprano.

Por el momento la información paleontológica disponible en la Formación Bajo Barreal en el área de estudio no permite asignar fehacientemente una edad CampanianoMaastrichtiano para estos niveles. En el caso de Aeolosaurus, existiría la posibilidad de que se trate de un linaje endémico no registrado aún antes del Campaniano-
Maastrichtiano, por lo que este género aisladamente no tendría un sustento cronológico fuerte. Pero el hallazgo de este titanosaurio derivado permite pensar en la posibilidad de hallar otros integrantes de la asociación faunística descrita en Los Alamitos por Bonaparte (1986, 1992), como los hadrosáuridos, que serían mejores indicadores cronológicos.

Con respecto a esto debemos mencionar al hadrosaurio Secernosaurus koerneri Brett Surman (1979) que, aunque tiene una procedencia estratigráfica dudosa, ya que se asigna a la Formación San Jorgé, inexistente en la cuenca del Golfo San Jorge, si es segura su procedencia geográfica del río Chico en el Sur de Chubut. Las observaciones de campo muestran que la única unidad estratigráfica continental del Cretácico aflorante en esa región es el miembro superior de la Formación Bajo Barreal. Esta falta de una base estratigráfica precisa, ha hecho que $S$. koerneri fuera excluido de diversos análisis bioestratigráficos. Además existe en la región el registro dudoso de una mandíbula asignada al ceratopsio Notoceratops bonarelli Tapia (1919). Ambos registros estarían vinculados al intercambio faunístico ocurrido al menos desde el Campaniano temprano a través de puentes geográficos entre América del Norte y América del Sur (Bonaparte et al., 1984; Bonaparte, 1986) junto con anquilosaurios (Salgado \& Coria, 1996), lagartos Teiidae y mamíferos Eutheria (Bonaparte, 1986). Como fauna emigrante hacia el norte se mencionan a saurópodos Titanosauridae (Bonaparte et al., 1984) y ofidios booideos (Rage, 1981; Albino, 1986).

Posiblemente los estudios que se están realizando sobre nuevos materiales extraídos en el SE del lago Colhué Huapi y nacientes del río Chico permitan ir perfilando una fauna más derivada que la registrada históricamente en la Formación Bajo Barreal y además aportar datos que permitan acotar la edad de estos afloramientos.

\section{CONCLUSIONES}

El material UNPSJB-PV 959 es incluido dentro del género Aeolosaurus, constituyendo así el registro más austral conocido de Aeolosaurini.

Se propone como nuevo carácter sinapomórfico del género Aeolosaurus la posición muy anterior de las postzigapófisis respecto del cuerpo vertebral en las caudales medias proximales (Figura 6).

Aeolosaurus colhuehuapensis sp. nov. sería el titanosaurio más derivado registrado hasta el momento en la fauna de la Formación Bajo Barreal.

La presencia de Aeolosaurus apoyaría la asignación de estos niveles del miembro superior de la Formación Bajo Barreal al Campaniano-Maastrichtiano?. Esto es coherente con las interpretaciones estratigráficas actuales de la región Sudeste del lago Colhué Huapi lo que se vería reforzado, en el futuro, con el hallazgo, estratigráficamente preciso, de otros integrantes de la asociación faunística definida por Bonaparte $(1986,1992)$ que tengan una clara implicancia cronológica, como los hadrosaurios. 


\section{AGRADECIMIENTOS}

Agradecemos a R. Espinoza y su equipo por dar aviso del hallazgo y por todo su apoyo logístico; a A. Horquera, M. Britapaja y D. González por su colaboración en los trabajos de extracción; a L. Ibiricu, E. Ivany, C. Navarrete y Y. Kamerbeek por su colaboración en los trabajos de laboratorio; a L. Salgado, R. Candeiro y R. Santucci por sus observaciones; a H. Pezzuchi por su apoyo desde la Secretaría de Ciencia y Técnica; y también a la Facultad de Ciencias Naturales de la Universidad Nacional de la Patagonia San Juan Bosco.

\section{REFERENCIAS}

Albino, A. 1986. Nuevos Boidae Madtsoiinae en el Cretácico Tardío de Patagonia (Formación Los Alamitos, Río Negro, Argentina). In: CONGRESO ARGENTINO DE PALEONTOLOGÍA Y BIOESTRATIGRAFÍA, 4, 1986. Actas, Mendoza, 2:63-95.

Bertini, R.J.; Santucci, R.M. \& Arruda-Campos, A.C. 1999a. First occurence of Aeolosaurus (Sauropoda, Titanosauridae) in Bauru Group of the Paraná Basin. Brazil. In: CONGRESSO BRASILEIRO DE PALEONTOLOGIA, 16, 1999. Boletim de Resumos, Crato, SBP, p. 27-28.

Bertini, R.J.; Santucci, R.M. \& Ribeiro, L.C.B. 1999b. O titanossáurido Aeolosaurus sp. (Saurischia, Sauropoda) no Membro Serra da Galga da Formação Marília. Grupo Bauru do Triângulo Mineiro. In: SIMPÓSIO DE GEOLOGIA DO SUDESTE, 6, 1999. Boletim de Resumos, São Pedro, UNESP, p. 78.

Bonaparte, J.F. 1986. History of the terrestrial Cretaceous vertebrates of Gondwana. In: CONGRESO ARGENTINO DE PALEONTOLOGÍA Y BIOESTRATIGRAFÍA, 4, 1986. Actas, Mendoza. 2:63-95.

Bonaparte, J.F. 1992. Una nueva especie de Triconodonta (Mammalia), de la Formación Los Alamitos, provincia de Río Negro y comentarios sobre su fauna de vertebrados. Ameghiniana, 29(2):99-110.

Bonaparte, J.; Franchi, M.; Powell, J.E. \& Sepúlveda, E. 1984. La Formación Los Alamitos (Campaniano-Maastrichtiano) del sudeste de Río Negro. Con descripción de Kritosaurus australis n. sp. (Hadrosauridae). Significado paleogeográfico de los vertebrados. Revista de la Asociación Geológica Argentina, 49(34): 284-299.

Brett Surman, M.K. 1979. Phylogeny and palaeobiogeography of hadrosaurian dinosaurs. Nature, 277(5697):560-562.

Calvo, J.O. \& Bonaparte, J.F. 1991. Andesaurus delgadoi gen. et sp.nov. (Saurischia-Saurópoda), dinosaurio Titanosauridae de la Formación Rio Limay (Albiano-Cenomaniano), Neuquén, Argentina. Ameghiniana, 28(3-4):303-310.

Calvo, J.O. \& González Riga, B. 2003. Rinconsaurus caudamirus gen. et sp. nov., a new titanosaurid (Dinosauria, Saurópoda) from the late Cretaceous of Patagonia, Argentina. Revista Geológica de Chile, 30(2):333-353.

Casal, G.; Luna, M.; Martínez, R.; Lamanna, M.; Sciutto, J.C. \& Ivany, E. 2006. La fauna Campaniana-Maastrichtiana? de la Formación Bajo Barreal en el E-SE del Lago Colhué Huapi, Provincia de Chubut, Argentina. In: JORNADAS ARGENTINAS DE PALEONTOLOGÍA DE VERTEBRADOS, 22, 2006. Resúmenes, San Juan, APA, p. 28.

Feruglio, E. 1949. Descripción Geológica de la Patagonia. Tomo
II, Dirección General de Yacimientos Petroliferos Fiscales, Buenos Aires, 349 p.

Franco-Rosas, A.C.; Salgado, L; Rosas, C. \& Souza Carvalho, I. 2004. Nuevos materiales (Saurópoda) en el Cretácico Superior de Mato Grosso, Brasil. Revista Brasileira de Paleontología, 7(3):329-336.

Gilmore, C.W. 1946. Reptilian fauna of the North Horn Formation of Central Utah. United States Geological Survey Prof. Papers, 210C:1-52.

González Riga, B. 2003. A new titanosaur (Dinosauria, Sauropoda) from the Upper Cretaceous of Mendoza Province, Argentina. Ameghiniana, 40(2):155-172.

Ibiricu, L.M. 2004. Identificación y estudio preliminar de un dinosaurio del Lago Colhué Huapi, en la Formación Bajo Barreal, Cretácico Superior del sur de Chubut. Universidad Nacional de la Patagonia San Juan Bosco, Tesis de Licenciatura inédita, 38 p.

Kellner, A.W.A. \& Azevedo, S.A.K. 1999. A new sauropod dinosaur (Titanosauria) from the Late Cretaceous of Brazil. In: GONDWANA DINOSAUR SIMPOSIUM, 2, 1999. Proceedings, Tokio, 15:111-142.

Kellner, A.W.A.; Campos, D.A. \& Trotta, M.N.F. 2005. Description of a Titanosaurid caudal series from the Bauru Group, Late Cretaceous of Brazil. Arquivos do Museu Nacional do Rio de Janeiro, 63(3):529-564.

Lamanna, M.; Luna, M. ; Casal, G.; Martínez, R.; Ibiricu, L. \& Sciutto, J.C. 2003. New Crocodyliform and Dinosaur Discoveries From The Upper Cretaceous (CampanianMaastrichtian?) Upper Member Of The Bajo Barreal Formation, Southern Chubut Province, Argentina. In: ANNUAL MEETING OF THE SOCIETY OF VERTEBRATE PALEONTOLOGY, 63, 2003. Abstract of Papers, Minnesota, SVP, p. $70 \mathrm{~A}$.

Luna, M.; Casal, G.; Martínez, R.; Lamanna, M.; Ibiricu, L. \& Ivany, E. 2003. La presencia de un Ornithopoda en el Miembro Superior de La Formación Bajo Barreal, Cretácico Superior (Campaniano-Maastrichtiano?) del Sur de Chubut. In: JORNADAS ARGENTINAS DE PALEONTOLOGÍA DE VERTEBRADOS, 19, 2003. Resúmenes, Buenos Aires, APA, p. 61.

Martínez, R. 1998. An articulated skull and neck of Sauropoda (Dinosauria: Saurischia) from the Upper Cretaceous of central Pategonia, Argentina. In: ANNUAL MEETING OF THE SOCIETY OF VERTEBRATE PALEONTOLOGY, 58, 1998. Abstract of Papers, Snowbird, SVP, p. 61A.

Martínez, R.; Giménez, O.; Rodríguez, J.; Luna, M. \& Lamanna, M. 2004. An articulated specimen of the basal Titanosaurian (Dinosauria: Saurópoda) Epachthosaurus sciuttoi from the Early Late Cretaceous Bajo Barreal Formation of Chubut Province, Argentina. Journal of Vertebrate Paleontology, 24(1):107-120.

Martínez, R.; Vita, J.; Lamanna, M. \& Ibiricu, L. 2006. A CT scan of a titanosauriform skull (Dinosauria: Sauropoda) from Central Patagonia, Argentina. In: ANNUAL MEETING OF THE SOCIETY OF VERTEBRATE PALEONTOLOGY, 66, 2006. Abstract of Papers, Ottawa, SVP, p. 96A.

Powell, J.E. 1986. Revisión de los Titanosauridos de América del Sur. Universidad Nacional de Tucumán, Tesis Doctoral, $340 \mathrm{p}$.

Powell, J.E. 1987. The Late Cretaceous Fauna from Los Alamitos, Patagonia, Argentina. Part. VI. The titanosaurids. Revista Museo Argentino Ciencias Naturales, 3(3):147-153.

Powell, J.E. 2003. Revision of South American Titanosaurid dinosaurs: palaeobiological, palaeobiogeographical and 
phylogenetic aspects. Records of the Queen Victoria Museum, 111:173 p.

Rage, I.C. 1981. Les continents peri-atlantiques au Cretacé Superieur: migrations des faunes continentales et problemes paleogeographiques. Cretaceous Research, 68: 457-494.

Salgado, L. \& Coria, R.A. 1993. El género Aeolosaurus (Sauropoda. Titanosauridae) en la Formación Allen (CampanianoMaastrichtiano) de la Provincia de Río Negro, Argentina. Ameghiniana, 30(2):119-128.

Salgado, L. \& Coria, R.A. 1996. First evidence of an ankylosaur (Dinosauria, Ornithischia) in South America. Ameghiniana, 33(4):367-371.

Salgado, L.; Coria, R.A \& Calvo, J.O. 1997. Presencia del género Aeolosaurus (Sauropoda, Titanosauridae) en la Formación Los Alamitos, Cretácico Superior de la Provincia de Río Negro, Argentina. Revista Universidade de Guarulhos, 2(6):44-49.
Salgado, L. \& García, R. 2002. Variación morfológica en la secuencia de vértebras caudales de algunos titanosaurios. Revista Española de Paleontología, 17(2):211-216.

Salgado, L.; García, R. \& Daza, J. 2006. Consideraciones sobre las láminas neurales de los dinosaurios saurópodos y su significado morfofuncional. Revista Museo Argentino Ciencias Naturales, 8(1):69-79.

Sciutto, J.C. 1981. Geología del codo del río Senguerr, Chubut, Argentina. In: CONGRESO GEOLÓGICO ARGENTINO, 8, 1981. Actas, San Luis, 3:203-219.

Sciutto, J.C.; Césari, O. \& Iantanos, N. en prensa. Hoja geológica 4566-IV Escalante, Escala 1:250000. Programa Nacional de Cartas Geológicas de la República Argentina, Secretaría de Minería de la Nación, Dirección Nacional del Servicio Geológico.

Tapia, A. 1919. Una mandíbula de dinosaurio procedente de Patagonia. Revista de la Sociedad Argentina de Ciencias Naturales, 4:369-370.

Received in September, 2006; accepted in March, 2007. 